\title{
An Inhomogeneous Structured Eversion Actuator ${ }^{\star}$
}

\author{
Taqi Abrar ${ }^{[0000-0002-8519-8356]}$, Ahmed Hassan ${ }^{[0000-0001-5368-8863]}$, Fabrizio \\ Putzu ${ }^{[0000-0001-7065-8025]}$, Hareesh Godaba ${ }^{[0000-0001-6600-8513]}$, Ahmad \\ Ataka $^{[0000-0003-3082-5778]}$, and Kaspar Althoefer ${ }^{[0000-0002-1141-9996]}$ \\ Centre for Advanced Robotics @ Queen Mary (ARQ), \\ Queen Mary University of London, Mile End Road, \\ London, E1 4NS, United Kingdom \\ (t.abrar, ahmed.hassan, f.putzu, h.godaba, a.rizqi, k.althoefer) \\ eqmul.ac.uk
}

\begin{abstract}
Soft actuators are free from any rigid, bulky, and hard components. This is greatly beneficial towards achieving compliant actuation and safe interactions in robots. Inspired by the eversion principle, we develop a novel soft actuator of the inhomogeneous cross-section that can linearly extend and achieve a large payload capability. The proposed soft actuator is a hollow sleeve, made from an airtight fabric, and features a top part of cylindrical shape and a bottom part of a conical shape. Unlike conventional eversion robots that extend unilaterally from the tip, in this proposed actuator the top cylindrical part and the bottom conical part are partially folded inwards so that the two tips are attached together. When pneumatic pressure is applied, the cylindrical part everts increasing in length while the conical section reduces in length folding inwards. The actuator achieves linear strains of $120 \%$ and can generate a force of $84 \mathrm{~N}$ at a low pressure of $62 \mathrm{kPa}$. We develop a theoretical model to describe the force and strain characteristics of the actuator during eversion from conical shape to cylindrical shape. The results showcase a step towards large strain, high force actuators for safe and compliant robots.
\end{abstract}

Keywords: Eversion actuator - Inflatable structure - Soft actuators · Design parameters.

\section{INTRODUCTION}

Soft robotics has gained enormous attention from the researchers resulting in developments of new kinds of soft actuators and robots in the recent era. These soft actuators overcome the barriers of their rigid counterparts in aspects such as safety, adaptability, and low cost. They have made a mark in many application

\footnotetext{
* This work was supported in part by the EPSRC National Centre for Nuclear Robotics project (EP/R02572X/1) and the Innovate UK WormBot project (104059).
} 
areas including grasping of delicate and fragile objects [1], wearable robots [2,3], and exploration of extreme environments that are not accessible by humans [4-6].

One of the main enablers of soft robots is the linear actuator that can generate translatory motion. Many linear actuators contract when pressurized by air such as pouch motors [7], McKibben muscles [8], and bio-inspired actuator [9,10], or extend in length such as the vine inspired actuator and robots [11,12], bioinspired inflatable actuator [13] and fiber reinforced actuator [14]. These actuators generally have a fixed end while the other end translates depending upon the actuation pressure causing the change in length of the actuator. The most important considerations of these expanding/contracting linear actuators are the actuation strain and the payload capability.

Eversion actuators are soft pneumatic actuators that are bio-inspired and perform linear actuation. They are generally cylindrical shaped and made from soft materials like plastics and fabrics [11]. The distal part of the cylindrical socklike structure is folded inside itself and the proximal opening is connected to a pneumatic input. When the air is pressurized inside the actuator, the previously folded part unfolds with the cylindrical structure gradually everting from the tip. This mechanism has led to achieving unprecedented extension ranges spanning tens of meters in length. Moreover, the lack of stretching in the material allows the optimal generation of axial forces due to an applied pressure [15].

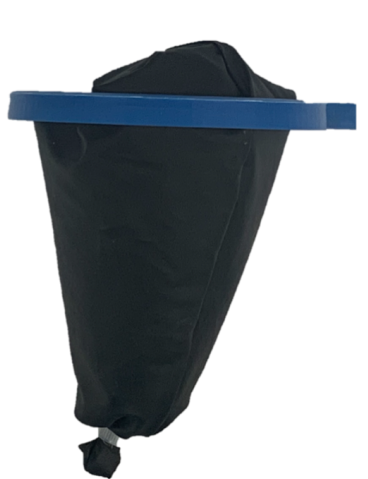

(a) Conical shape in the uninflated state

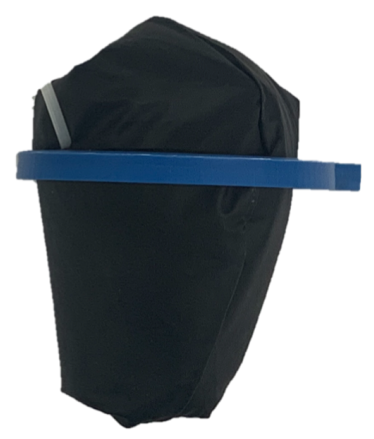

(b) Intermediate state

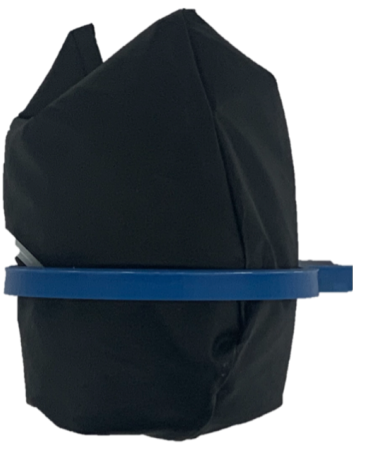

(c) Cylindrical shape in the fully inflated state

Fig. 1: Actuation and geometrical changes in the actuator (a) when the actuator is uninflated, it is conical in shape, (b) when actuated, the cylindrical part everts and the conical tip draws in towards the centre of the actuator, (c) cylindrical part is completely everted and the cone is completely inverted. 
In this paper, we propose a new kind of soft pneumatic actuator inspired by the eversion mechanism (Figure 1). The actuator has an inhomogeneous structure, the bottom half has a conical shape (Figure 1a) while the top half part of the actuator has a cylindrical shape (Figure 1c). The actuation mechanism of the actuator is based on the eversion principle. This design allows the actuator to achieve stroke length of up to $100 \%$ tip displacement which is higher than the actuation strains in many of the existing pneumatic soft actuators such as pouch actuators and McKibben's muscles. Furthermore, there are minimal energy losses due to stretching of material or due to friction, as the outer segment of the eversion actuator does not slide against the external environment.

The remainder of this paper is organized as follows: The geometry and fabrication of the actuator are discussed in Section 2. In Section 3, we develop an analytical model that can predict the actuation characteristics. Experimental results and simulation studies are presented in Section 4, followed by discussion and future work in Section 5.

\section{HARDWARE DESIGN}

An actuator that extends its length by everting out its body from the center when pressurised can be categorised as an eversion actuator [16]. They are made from soft materials and are lightweight, compliant in nature, and can squeeze through narrow regions [10]. They also have low energy losses due to the friction and can grow from a very small size to multiple times of their initial length $[12,16]$.

The traditional eversion-based actuators or robots in the literature have a uniform cylindrical cross-section with one side connected to the air source for pressurizing the actuator [12]. In an attempt to develop a fully self-contained actuator based on the eversion principle, both ends of the cylindrical tube can be folded inwards and connected at the center. However, in this configuration, a pressure applied to the inner chamber of the structure will only cause it to stiffen without producing any displacement. To solve this problem, we create the actuator from a profile of inhomogeneous cross-section. This will enable the actuator to produce eversion (unfolding of material) on one end and inversion (folding of the material) at the other end of the actuator, thereby causing a displacement and actuation force. The design and the construction of such an inhomogeneous eversion actuator is presented in this section.

\subsection{Design of the actuator}

The actuator consists of two different geometrical shapes combined together. The top part is cylindrical and the bottom part is conical, which makes the whole structure of the actuator inhomogeneous. For the construction of the actuator, an airtight fabric sheet (Breathable Coated Microfibre from UKfabrics Online) is cut in the desired shape (Figure 2). As shown in the figure, the cross-section of the actuator is made to be inhomogeneous by attaching the top of the cone to the bottom of the cylindrical shape. The two adjacent longitudinal sides are 


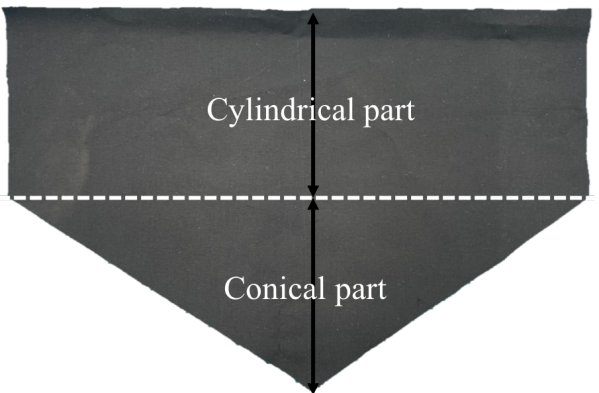

Fig. 2: Fabrication of the actuator: a flat sheet is cut into the shape shown above to form a cylindrical and a conical part.

sewn to construct the desired shape of the actuator. To ensure that there is no air leakage, all the seams and closures are sealed using a cyanoacrylate super glue. Once the sealing is done, the structure is turned inside out, moving the created seams to the inside of the actuator.

\subsection{Eversion in the proposed inhomogeneous actuator}

To complete the fabrication process, the tip of the cone (which is at the bottom) is folded inward and attached to the closed end of the cylinder (which is at the top). Once the tip of the cone and the cylinder are joined together and sealed, the tip is pulled back such that the cylinder now rests inside the conical structure. As a result, the shape of the visible actuator becomes conical with the cylindrical part folded inside. When pneumatic pressure is applied during actuation, the previously folded part moves in the direction away from the bottom (the tip of the cone), achieving a movement based on the principle of eversion (Figure 1b).

As illustrated in Figure 3, the tip of the cone is attached to the top of the cylindrical shape. When the previously folded part unfolds while everting from the top of the cone, the top of the cylinder pulls the tip of the cone. In other words, when the cylindrical part of the actuator is everting, the conical part inverts and folds into the actuator. This results in an increase in the length of one (cylindrical) side and simultaneously, reduction in the length of the other (conical) side. As a consequence, the total length of the actuator remains fixed, but the tip of the actuator is displaced across the whole length of the actuator.

\section{ANALYTICAL MODEL}

We now develop an analytical model to describe the forces and actuation strains in the actuator when it is actuated by pneumatic pressure. At the initial unactuated state (also called the reference state), the geometry of the actuator is given by dimensions of the cone- radius, $r_{c o}$ and height, $h_{c o}$. At any given state 


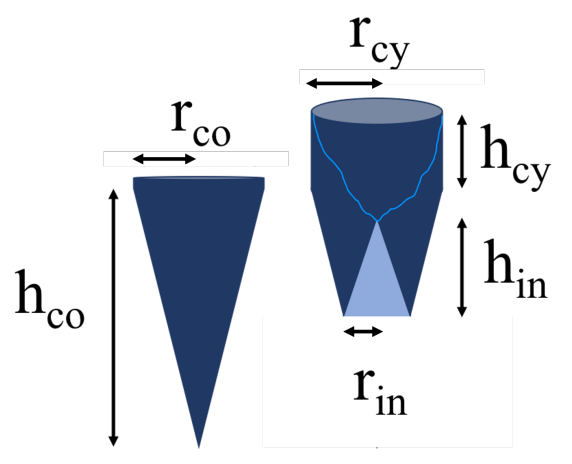

Fig. 3: Geometry of the inhomogeneous eversion actuator in the reference (unactuated) state and an actuated state.

of the actuator, its geometry can be represented by the everted length, $h_{c y}$, the inverted length, $h_{i n}$, and the radius of the cross-section at the bottom tip in the conical part, $r_{i n}$ as shown in Figure 3.

The bottom of the actuator is composed of conical shape and when the actuator is not inflated, the shape of the actuator will be conical, therefore the volume of the actuator at the beginning can be defined as;

$$
V_{c o}=\frac{\pi r_{c o}^{2} h_{c o}}{3}
$$

Where $V_{c o}$ is the volume of the cone, $r_{c o}$ is the radius of the cone, and $h_{c o}$ is the height of the cone. While on the other hand, when fully-inflated, only the top part (which is cylindrical in shape) is visible, therefore the volume of the actuator when it is fully extended can be defined as

$$
V_{c y}=\pi r_{c y}^{2} h_{c y}
$$

Where $V_{c y}$ is the volume of the cylinder, $r_{c y}$ is the radius of the cylinder, and $h_{c y}$ is the height of the cylinder. While the top part is everting, at the same time, the tip of the cone (bottom of the actuator) starts inverting. The volume of the cone $V_{\text {in }}$ inverted at any state can be defined as

$$
V_{i n}=\frac{\pi r_{i n}^{2} h_{i n}}{3}
$$

Where $r_{i n}$ is the radius of the cone and $h_{i n}$ is the height of the cone folded back due to the eversion at the top part of the actuator. Since the volume and shape of the actuator is changing from conical to cylindrical, therefore the volume of the actuator $V_{a}$ any point of time can be defined as

$$
V_{a}=V_{c o}-2 V_{i n}+V_{c y}
$$


By substituting the values in above the equation 4, the new equation 5 will be

$$
V_{a}=\frac{\pi r_{c o}^{2} h_{c o}}{3}-\frac{2 \pi r_{i n}^{2} h_{i n}}{3}+\pi r_{c y}^{2} h_{c}
$$

The above equation 5 contains 2 types of parameters, constant dimensions and variable parameters.

\begin{tabular}{||c|c||}
\hline Constant dimensions & Variable parameters \\
\hline \hline$r_{c o}$ & $r_{i n}$ \\
\hline$h_{c o}$ & $h_{i n}$ \\
\hline$r_{c y}$ & $h_{c y}$ \\
\hline
\end{tabular}

Based on the geometry,

$$
\begin{gathered}
r_{c o}=r_{c y}=r_{a}, \\
h_{c y}=h_{i n}=h_{e},
\end{gathered}
$$

Where $r_{a}$ is the radius of the actuator and $h_{e}$ is the height of the eversion. Therefore the proposed actuator has two knowns (fixed) and two unknowns (variable) parameters.

\begin{tabular}{||c|c||}
\hline Fixed parameter & Variable parameters \\
\hline \hline$r_{a}$ & $r_{i n}$ \\
\hline$h_{c o}$ & $h_{e}$ \\
\hline
\end{tabular}

From the geometry of the cone, we can also estimate the relationship between the conical shape of the actuator and the cone which goes inside the actuator during eversion.

$$
\frac{h_{e}}{r_{i n}}=\frac{h_{c o}}{r_{a}}
$$

With this relationship the equation 5 will become

$$
\begin{gathered}
V_{a}=\frac{\pi}{3}\left(r_{a}^{2} h_{c o}-2 r_{i n}^{2} h_{e}+3 r_{a}^{2} h_{e}\right) \\
V_{a}=\frac{\pi}{3}\left(r_{a}^{2} h_{c o}-2 \frac{r_{a}^{2} h_{e}^{2} h_{e}}{h_{c o}^{2}}+3 r_{a}^{2} h_{e}\right) \\
V_{a}=\frac{\pi r_{a}^{2}}{3}\left(h_{c o}-2 \frac{h_{e}^{3}}{h_{c o}^{2}}+3 h_{e}\right) \\
V_{a}=\frac{\pi r_{a}^{2} h_{c o}}{3}\left\{1-2\left(\frac{h_{e}}{h_{c o}}\right)^{3}+\frac{3 h_{e}}{h_{c o}}\right\} \\
V_{a}=V_{c o}\left\{1-2\left(\frac{h_{e}}{h_{c o}}\right)^{3}+\frac{3 h_{e}}{h_{c o}}\right\}
\end{gathered}
$$


Differentiating equation 13 with respect to $h_{e}$, we get;

$$
\frac{d V_{a}}{d h_{e}}=\left(-\frac{6 V_{c o} h_{e}^{2}}{h_{c o}^{3}}+\frac{3 V_{c o}}{h_{c o}}\right)
$$

Due to the conservation of energy, the work done by the actuator by the virtual displacement of a load is equal to the work done by the air pressure through a change in volume of the actuator. This relation is given as;

$$
W=-F d h=P d v .
$$

So, the actuating force is given by;

$$
F=-P \frac{d V_{a}}{d h_{e}}
$$

Substituting the value of $d V_{a} / d h_{e}$ from equation 14 in above equation 16 , we get:

$$
F_{h_{e}}=-\frac{3 P V_{c o}}{h_{c o}}\left\{1-2\left(\frac{h_{e}}{h_{c o}}\right)^{2}\right\}
$$

\section{RESULTS}

We conduct experiments to understand the payload capabilities of the actuator at different isometric strains. The pressure applied to the actuator is regulated via an electro-pneumatic regulator (SMC-ITV2050-212BL4). The actuator is in the state where the top part is folded inside (see Figure 1a) and attached to the bottom part (the tip of the cone) as shown in Figure 3. The bottom of the cone is attached to the Z-axis of the RFT Force Torque sensor (ROBOTOUS RFT40-SA01-D) to measure the force exerted by the actuator on an object at zero actuation strain.

An actuator with the following representative dimensions was built. The conical part has a length of $100 \mathrm{~mm}$ while having a radius of $37.5 \mathrm{~mm}$ and the cylindrical part has the same radius of $37.5 \mathrm{~mm}$ and the same active length of $100 \mathrm{~mm}$.

\subsection{Blocked Force Test}

In this section, the experiment was conducted to measure the maximum force generation capability of the actuator. The actuator is attached to a rigid $3 \mathrm{~d}$ printed structure which is firmly affixed to a support. The tip of the cone of the actuator in the reference configuration (figure 1a) is attached to a fixed force sensor. This configuration also corresponds to the zero strain configuration of the actuator. Different pneumatic pressures ranging from 0 to $69 \mathrm{kPa}$ in increments of $6.9 \mathrm{kPa}$ were applied to the actuator and the forces measured by the FT sensor are noted. The maximum test pressure is limited to $69 \mathrm{kPa}$ to prevent fracture of 
the actuator. When pressure is applied, the cylindrical fabric inside the conical region tries to evert and applies force on the tip which pulls on the force sensor. Consequently, a force is recorded by the FT sensor. From figure 4, we see that as the pressure increases, the force increases almost linearly with pressure showing a piston type behaviour.

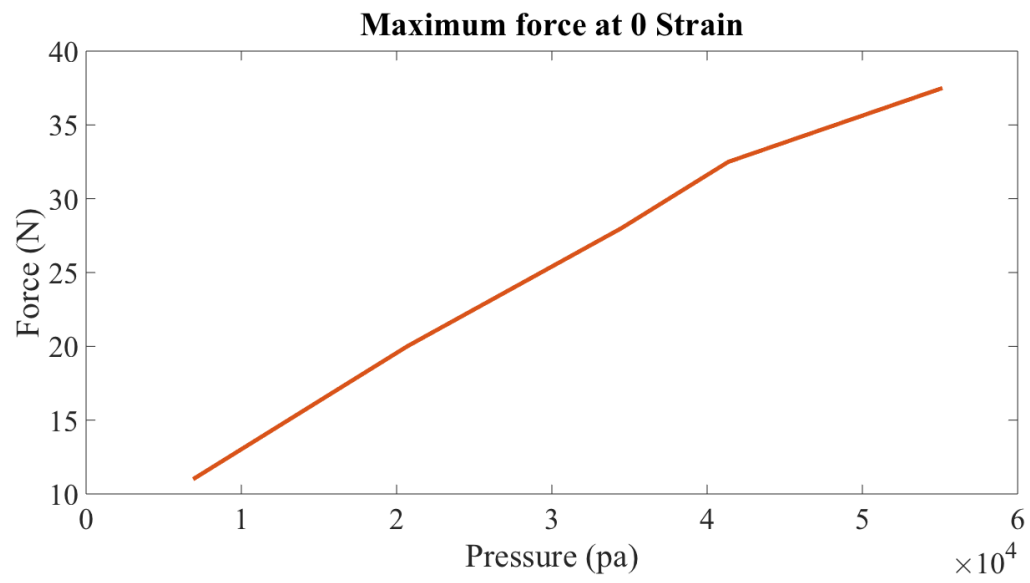

Fig. 4: Blocked force test results. Force exerted by the actuator when attached to a fixed FT (force-torque) sensor

In the proposed model (equation 17), the force has a linear relationship which agrees well with the experimental measurements for the force generated by the actuator (Figure 4). Further increase in the pressure can increase the force generated by the actuator but may cause a fracture when the stresses in the fabric reach the yield strength of the material. The seams sealed by adhesive are also prone to leakage at high pressures.

\subsection{Actuation force at different strains}

Unlike a piston in a cylinder, when the eversion actuator is undergoing actuation, the effective cross-section area of the everting tip keeps changing due to the varying cross-section caused by the cylinder in cone design. Hence, the payload capability of the actuator varies with the actuation strain. To understand this variation, we conduct experiments to measure the force output of the actuator under a fixed pressure at varying strains. The frame to which the base of the actuator (seen as the blue annular frame in Figure 5) is mounted on the Instron universal testing machine (UTM) fitted with a $2 \mathrm{kN}$ load cell as shown in Figure 5. The load cell is attached to the tip of the cone via a fishing line acting as an inextensible thread. The Instron head is programmed to translate through $100 \mathrm{~mm}$ stroke length with a speed of $1 \mathrm{~mm} / \mathrm{s}$. 


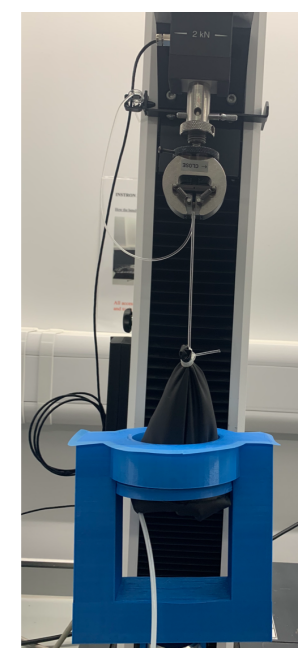

Fig. 5: Inhomogeneous eversion actuator mounted on a universal testing machine (UTM) for characterizing the generated forces

Three different pressures are applied to the actuator and the pull tests are conducted. Figure 6a shows that the response of the actuator. When the input pressure is increased, the magnitude of force generated by the actuator also increases. At a pressure of $62 \mathrm{kPa}$, force as high as $80 \mathrm{~N}$ is produced. We can see that, as the strain increases to the maximum, the force in all three pressure cases reduces to zero. The scaling of the forces for a given strain is nearly proportional to the pressure reinforcing observation noted in the analytical model.

\subsection{Theoretical Results}

We now utilise the model developed in Section 3 to calculate the actuation force at different strains and compare them to the experimental results. Figure $6 \mathrm{~b}$ illustrates the force required by the actuator to create a specified strain for a given input pressure. As the strain increases to the maximum, the force decreases to zero linearly. This is qualitatively similar to the experimental results in Figure 6a. The predicted values for the force are higher than the experimental values due to several reasons. We assume no pressure loss due to leakage while in the experiments, there is slight leakage from the seams and the actuator is considered to be made of an ideal cylinder and a cone that may deviate from the experiments.

In a practical scenario, when the actuator is used as an artificial muscle, it sometimes needs to pull or push the constant load, for example, lift a mass suspended from the tip of the actuator. To model this scenario, the effect of the payload on the actuation strain at a given pressure in the previously discussed pressure range of $0-69 \mathrm{kPa}$ with increments of $6.9 \mathrm{kPa}$, is calculated. Different 


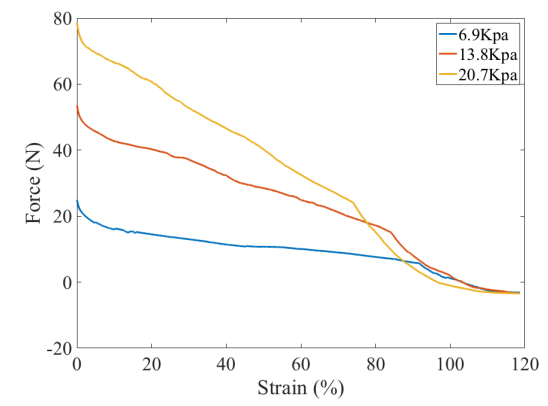

(a) Relationship of pressure against strain

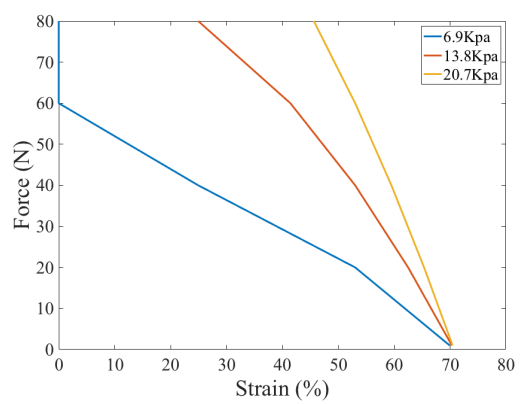

(b) Relationship of strain vs force

Fig. 6: Performance of the actuator. (a) Measured force as a function of strain for different applied pressures.(b) Modelled Force as a function of strain for different applied pressures.

loads ranging from no load to a load of $30 \mathrm{~N}$ with increments of $5 \mathrm{~N}$ are considered. Figure 7 shows the strain achieved as a function of pressure with different constant loads applied at the tip. This is analogous to the practical case in which a load is attached to the tip of a vertically aligned actuator and pressure is applied to the actuator to lift the load. We can see that when the load is small, the actuator can lift the load to its maximum stroke length at a low pressure. However, when the load is increased it requires higher pneumatic pressure to lift the load to its maximum strain level. The non-linear nature of the pressure-strain curve is attributed to the geometry of the actuator and the non-linear nature of change of the volume of the actuator with the tip displacement.

\section{CONCLUSION}

The actuator based on the eversion of an inhomogeneous structure is presented in this paper. The inhomogeneous eversion actuator is capable of displacement up to $100 \%$ of its length from its original position. During the eversion and displacement of its tip, it can generate a significant payload. The actuator is constructed from fabric ensuring the actuator remains soft in nature. The inhomogeneous structure of the actuator is divided into two sections; the top portion which is initially folded inside is cylindrical in shape and everts from the bottom part which is conical in shape. Even made from soft material, this eversion based inhomogeneous actuator can fully actuate for a range of loads. A theoretical model to explain the mechanism of actuation is developed and the results are qualitatively consistent with the experiments. The actuator achieves a high force of over $80 \mathrm{~N}$ at a low pressure of $62 \mathrm{kPa}$. In future work, this actuator will be integrated into articulated mechanisms to achieve low weight and high payload compliant robots. 


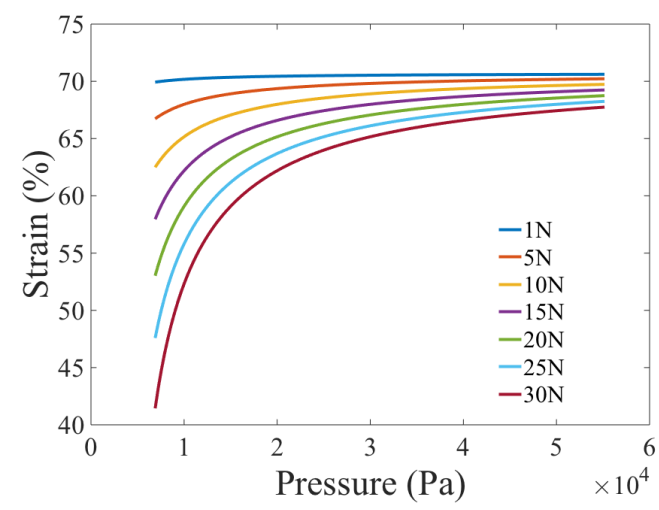

Fig. 7: Modelling result. Strain as a function of pressure for different applied constant loads.

\section{ACKNOWLEDGMENT}

This work was supported in part by the EPSRC National Centre for Nuclear Robotics project (EP/R02572X/1), the Innovate UK WormBot project (104059).

\section{References}

1. Hassan A, Godaba H, Althoefer K. Design Analysis of a Fabric Based Lightweight Robotic Gripper. InAnnual Conference Towards Autonomous Robotic Systems 2019 Jul 3 (pp. 16-27). Springer, Cham.

2. Abrar T, Putzu F, Althoefer K. Soft Wearable Glove for Tele-Rehabilitation Therapy of Clenched Hand/Fingers Patients. In Annual Conference on New Technologies for Computer and Robot Assisted Surgery 2018 Sep 11 (pp. 93-94).

3. Putzu F, Abrar T, Althoefer K. Development of a Soft Inflatable Structure with Variable Stiffness for Hand Rehabilitation. In Annual Conference on New Technologies for Computer and Robot Assisted Surgery 2018 Sep 11 (pp. 103-104).

4. Coad MM, Blumenschein LH, Cutler S, Zepeda JA, Naclerio ND, El-Hussieny H, Mehmood U, Ryu JH, Hawkes EW, Okamura AM. Vine Robots: Design, Teleoperation, and Deployment for Navigation and Exploration. arXiv preprint arXiv:1903.00069. 2019 Feb 28.

5. Luong J, Glick P, Ong A, deVries MS, Sandin S, Hawkes EW, Tolley MT. Eversion and Retraction of a Soft Robot Towards the Exploration of Coral Reefs. In2019 2nd IEEE International Conference on Soft Robotics (RoboSoft) 2019 Apr 14 (pp. 801-807). IEEE.

6. Ataka A, Stilli A, Konstantinova J, Wurdemann HA, Althoefer K. Kinematic Control and Obstacle Avoidance for Soft Inflatable Manipulator. InAnnual Conference Towards Autonomous Robotic Systems 2019 Jul 3 (pp. 52-64). Springer, Cham.

7. Niiyama R, Rus D, Kim S. Pouch motors: Printable/inflatable soft actuators for robotics. In2014 IEEE International Conference on Robotics and Automation (ICRA) 2014 May 31 (pp. 6332-6337). IEEE. 
8. Tondu B, Lopez P. The McKibben muscle and its use in actuating robot-arms showing similarities with human arm behaviour. Industrial Robot: An International Journal. 1997 Dec 1;24(6):432-9.

9. Maghooa F, Stilli A, Noh Y, Althoefer K, Wurdemann HA. Tendon and pressure actuation for a bio-inspired manipulator based on an antagonistic principle. In2015 IEEE International Conference on Robotics and Automation (ICRA) 2015 May 26 (pp. 2556-2561). IEEE.

10. Stilli A, Wurdemann HA, Althoefer K. Shrinkable, stiffness-controllable soft manipulator based on a bio-inspired antagonistic actuation principle. In2014 IEEE/RSJ International Conference on Intelligent Robots and Systems 2014 Sep 14 (pp. 24762481). IEEE.

11. Abrar TA, Putzu F, Konstantinova J, Althoefer K. EPAM: Eversive Pneumatic Artificial Muscle. In2019 2nd IEEE International Conference on Soft Robotics (RoboSoft) 2019 Apr 14 (pp. 19-24). IEEE.

12. Hawkes EW, Blumenschein LH, Greer JD, Okamura AM. A soft robot that navigates its environment through growth. Science Robotics. 2017 Jul 19;2(8):eaan3028.

13. Cianchetti M, Ranzani T, Gerboni G, Nanayakkara T, Althoefer K, Dasgupta P, Menciassi A. Soft robotics technologies to address shortcomings in today's minimally invasive surgery: the STIFF-FLOP approach. Soft robotics. 2014 Jun $1 ; 1(2): 122-31$.

14. Hawkes EW, Christensen DL, Okamura AM. Design and implementation of a 300\% strain soft artificial muscle. In2016 IEEE International Conference on Robotics and Automation (ICRA) 2016 May 16 (pp. 4022-4029). IEEE.

15. Godaba, H., Putzu, F., Abrar, T., Konstantinova, J. and Althoefer, K., 2019, July. Payload capabilities and operational limits of eversion robots. In Annual Conference Towards Autonomous Robotic Systems (pp. 383-394). Springer, Cham.

16. Putzu F, Abrar T, Althoefer K. Plant-Inspired Soft Pneumatic Eversion Robot. In2018 7th IEEE International Conference on Biomedical Robotics and Biomechatronics (Biorob) 2018 Aug 26 (pp. 1327-1332). IEEE. 